\title{
Gerenciador de Pílulas Inteligente
}

\author{
Mariane Kaviski \\ Universidade Tecnológica Federal do \\ Paraná \\ Curitiba, Paraná, Brasil \\ makaviski@gmail.com
}

\author{
Pedro Felipe Taveira dos \\ Santos \\ Universidade Positivo \\ Curitiba, Paraná, Brasil \\ eng_pedrosantos@outlook.com
}

\author{
Wilian Galezzi \\ Universidade Positivo \\ Curitiba, Paraná, Brasil \\ wgaleazzi@hotmail.com
}

\author{
Verônica Isabela Quandt \\ Universidade Positivo \\ Curitiba, Paraná, Brasil \\ veronica.quandt@gmail.com
}

\author{
Eduardo Juliano Alberti \\ Universidade Positivo \\ Curitiba, Paraná, Brasil \\ eduardojalberti@gmail.com
}

\begin{abstract}
In Brazil, as in other countries in the world, the percentage of elderly people has been increasing in recent decades. This fact is mainly caused by the drop in the birth rate and the increase in the average life span. As a result, health care for the elderly becomes increasingly important. Adherence to drug treatment is a clinical challenge for doctors who serve this population. Very old people have multiple illnesses and, consequently, they use multiple medications. Some also have impaired cognitive or sensory functions, such as visual pathologies or decreased motor skills. However, nonadherence to drug treatment can lead to the emergence of new diseases, hospitalizations or even death. This article presents the development of an intelligent device that has the function of assisting drug management, seeking the well-being of the elderly. The device allows the registration of the time and interval of medication intake through a list of products, regulated by Anvisa, pre-registered in the system and selected according to its format. After audible and visual warnings, the medication is dispensed automatically, allowing the medication to be ingested at the appropriate time. It is also possible to view a drug consumption report and registration times. After testing, the developed prototype was effective for the purpose for which it was designed. The encoder system, responsible for monitoring and controlling the position of the medication compartment disc, presented an average deviation of $0.0263 \mathrm{~mm}$, which prevents the overlapping of pills. At the same time, samples of medication availability at the scheduled times showed a zero error rate.
\end{abstract}

\section{KEYWORDS}

Dispensador de Pílulas, Idosos, Tecnologia

\section{INTRODUÇÃO}

A Organização Mundial da Saúde (OMS) recomenda para países em desenvolvimento que a população idosa corresponda a pessoas de 60 anos ou mais de idade, definição adotada pelo Instituto Brasileiro de Geografia e Estatística (IBGE) [5].

O envelhecimento da população está ocorrendo em todo o mundo. Este fato provém de fatores como mudanças no estilo de vida, diminuição das taxas de fecundidade e avanços dos recursos médicos e tecnológicos [6].

No Brasil, a proporção de pessoas de 60 anos ou mais de idade na população total foi de $10 \%$ em 2010, o que correspondia a 19,7 milhões de idosos. Em 2017, a Organização das Nações Unidas (ONU) realizou a revisão de projeção da população. Segundo esta projeção, em 2033 a proporção de idosos será de $20 \%$ da população total, correspondendo a 46 milhões de pessoas [5].

Segundo Rozendeld, idosos consomem em média de dois a cinco medicamentos ao dia, sendo que aproximadamente um terço desta população consome cinco ou mais medicamentos ao dia [3]. Devido a estes fatos, a gestão de medicamentos é um assunto relevante entre cuidadores de idosos e familiares. A manutenção e administração de medicamento de uso contínuo pode se tornar uma tarefa complexa à medida que o número de medicamentos aumenta.

Neste contexto, pela aplicação da tecnologia, pretende-se gerar melhor qualidade de vida de pessoas idosas, de seus cuidadores de familiares por meio de um dispositivo inteligente que tem como função auxiliar na gestão medicamentosa.

O dispositivo permite o cadastro do horário e intervalo de ingestão dos medicamentos através de uma lista de produtos, regulados pela Anvisa, pré-cadastrados no sistema e selecionados conforme seu formato. Após avisos sonoros e visuais, dispensa o medicamento de forma automática, possibilitando a ingestão do medicamento no horário adequado. Também é possível a visualização de um relatório de consumo dos medicamentos e dos horários de cadastro.

\section{TRABALHOS RELACIONADOS}

O primeiro dispensador de medicamentos com recursos de automação e eletrônica a ser comercializado no mundo foi o Dispensador Automático de Pílulas (DAP). É um dispositivo eletrônico motorizado desenvolvido pela Medinet Clinic, na Espanha, e comercializado através de distribuidores de loja física e loja virtual. Possui função de armazenamento de pílulas e alarmes pré-programados com os horários para ingestão delas. O dispensador DAP permite o fornecimento de medicamentos de forma controlada em até 6 programas diários. Contém 28 compartimentos individuais, onde são armazenados os conjuntos de medicamentos a serem ingeridos em cada horário especificado. A programação é simples e só é necessário fazê-la uma vez, desde que os horários não sejam modificados [2].

Em 2012, alunos de Engenharia da Computação do Centro Universitário de Rio Preto (UNIRP) desenvolveram um Dispensador 
Automático de Medicamentos. Consiste em um disco com 24 compartimentos, controlado por um motor de passo, onde são armazenados comprimidos e nos horários programados são disponibilizados para ingestão. Quando não há a retirada da pílula, o dispositivo alerta o cuidador via SMS. Diferente do DAP, o Dispensador Automático de Medicamentos consiste em uma inteligência onde não há necessidade de inserir os medicamentos de forma sequencial, o próprio dispositivo gerencia os medicamentos armazenados [1].

Um dos projetos mais recente é o Dispensador eletrônico de medicamentos (DIME), desenvolvido em 2016 por alunos do curso de Design de produto da Universidade Federal do Rio Grande do Sul (UFRGS). É um dispensador eletrônico de medicamentos para idosos portadores da doença de Alzheimer em estágio inicial de demência. Visa auxiliar o idoso a administrar sua medicação, permitindolhe autonomia, auxiliando cuidadores e familiares a organizar e controlar o uso da medicação [4].

\section{SOLUÇÃO PROPOSTA}

O sistema desenvolvido é capaz de gerenciar e armazenar pílulas, comprimidos ou cápsulas, alertando e gerenciando os horários em que usuários devem ingerir os medicamentos. $\mathrm{O}$ armazenamento consiste em espaços individuais para cada pílula em um disco, junto a sensores de encoder. Este tem como propósito a precisão do posicionamento de cada espaço de armazenagem, bem como monitorar se o local de armazenagem está vazio ou ocupado. A Figura 1 apresenta um diagrama de blocos dos módulos do sistema.

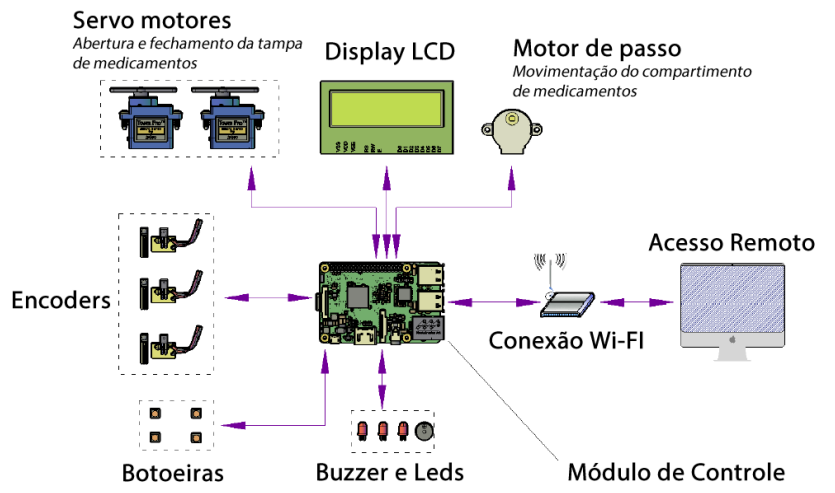

Figura 1: Diagrama em blocos dos módulos do sistema

O gerenciamento é realizado através do controle de quantidade de compartimentos ocupados por pílulas e da liberação para sua ingestão nos horários específicos. A parametrização é feita através de um display acoplado no próprio equipamento, no qual o usuário programa os horários de cada medicamento. É possível também visualizar relatórios dos horários de ingestão pela web. O dispositivo será de uso fixo, sendo necessário estar ligado à rede elétrica e ao sistema de internet para comunicação.

\subsection{Software}

O microcomputador Raspberry Pi 2 atua como módulo principal realizando a comunicação entre os componentes, a comunicação com a web e o controle de todos os motores contidos no projeto.
O desenvolvimento da programação e criação do código fonte do Gerenciador de Pílulas Inteligente foi realizado através do software Geany direto no Raspbian, sistema de operação da Raspberry Pi 2, utilizando linguagem $\mathrm{C}$ e $\mathrm{C}++$.

A Raspberry Pi 2 integra os módulos do projeto quando o usuário utiliza os módulos de botoeiras e display LCD para programar um novo medicamento. Quando um medicamento é inserido, é armazenada as informações de horário em que deve ser ingerido, posição e nome do medicamento, disponibilizando esses dados na web.

\subsection{Automação}

Para automação do sistema foi utilizado um conjunto de sensores e motores. Para rotacionar e controlar a posição do disco de compartimentos, local de armazenamento dos medicamentos, utilizou-se um motor de passo modelo $28 \mathrm{BYJ}-48$ associado a um encoder. $\mathrm{O}$ controle do motor de passo é feito através do circuito integrado ULN2003.

Quando o disco de compartimentos se encontra na posição correta de acordo com a programação, o módulo de sistema audiovisual é acionado pela RaspBerryPi 2, até que o botão solicitado seja acionado, iniciando a rotina de abertura do alçapão, através de dois servo motores modelo SG-90.

\subsection{Montagem Eletromecânica}

A Figura 2 representa o conjunto de dispositivos envolvidos quando há movimento no motor de passo. O disco de encoder e o disco de compartimentos devem rotacionar quando há trabalho no motor. Já os sensores de infravermelho e a camada de alçapões se mantém estáticos.

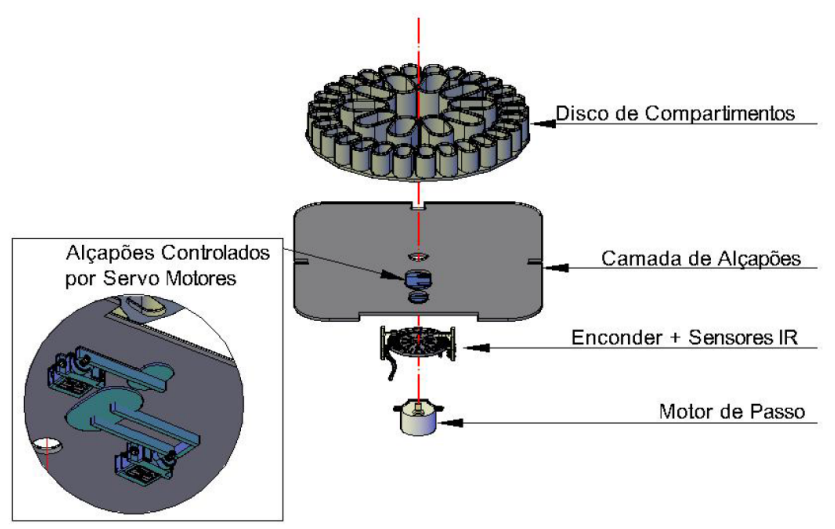

Figura 2: Diagrama da Montagem Eletromecânica

O Gerenciador Inteligente de Pílulas foi desenvolvido de modo a disponibilizar medicamentos, sejam estes em formato de pílulas, comprimidos ou cápsulas. Verificou-se então a necessidade de avaliar as dimensões utilizadas pela indústria farmacêutica para medicamentos.

Consequentemente, o disco de compartimentos possui dois modelos de compartimentos de pílulas. Foram dimensionadas 10 seções para medicamentos maiores com dimensões de $3,0 \mathrm{~mm}$ por $1,5 \mathrm{~mm}$, para capsulas do tipo 0 a 000 , que necessitam de espaço físico maior, 
localizadas no centro do disco. Para os demais tipos de medicamentos, foram projetadas 28 seções com dimensões de $1,8 \mathrm{~mm}$ por $1,0 \mathrm{~mm}$, alocadas no arranjo de compartimentos com raio mais próximo a margem do disco.

Abaixo do disco de compartimentos foi colocada a camada de alçapões, com tamanhos de abertura correspondentes às duas dimensões dos compartimentos do disco, com uma margem de segurança de $1 \mathrm{~mm}$ maior que estes.

Os servomotores realizam a operação de abertura e fechamento dos alçapões, sua rotina é iniciada no software quando é chegada a hora de ingestão de um comprimido. O acionamento do Micro Servo é realizado através de um sinal de PWM proveniente do Raspberry Pi 2. Este sinal de controle é aplicado ao pino de PWM do motor, que por sua vez recebe o sinal e interpreta deslocando o motor para a posição correspondente.

O sistema encoder é composto por um disco encoder e módulo sensor de obstáculo infravermelho. $\mathrm{O}$ disco projetado especificamente para este projeto possui $4 \mathrm{~cm}$ de diâmetro com 38 furos distribuídos conforme as seções do disco de compartimentos. $\mathrm{O}$ modulo sensor é um circuito formado por um emissor de infravermelho modelo SIM-22STF e um receptor fototransistor RPM-22PB, que se conectam com a Raspberry Pi 2 através de uma porta GPIO.

A lógica de funcionamento e rotação do motor de passo é estabelecido a partir de um software composto por uma matriz $4 \times 4$, onde cada linha da matriz corresponde a uma sequência para alimentar as bobinas do motor de passo. Cada bobina é alimentada por uma saída diferente do Raspberry Pi 2. Cada vez que é enviada uma sequência diferente da matriz para o conjunto do motor é realizado um passo. Quando é enviado um sinal do Raspberry Pi 2, o primeiro bloco atuante no conjunto do motor de passo é o drive ULN2003, um conversor de sinais de comando para sinais de potência, necessários para funcionamento do motor.

\subsection{Interface com o usuário}

Por meio de 4 botões e o display LCD, mostrados na Figura 3 o usuário acessa e ajusta os parâmetros. Cada botão está conectado ao Raspberry Pi 2 através de uma porta GPIO.

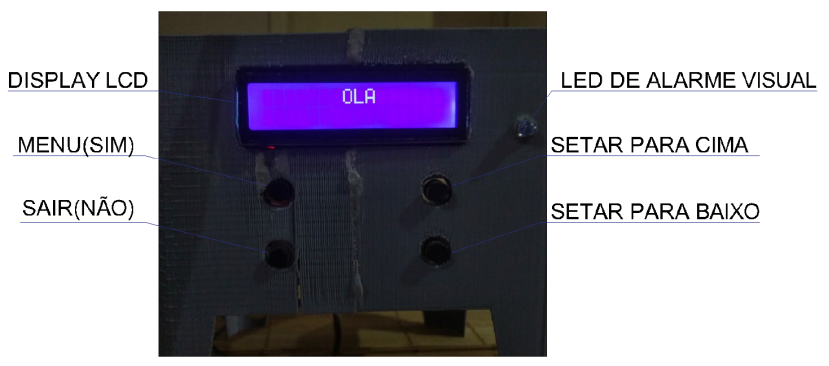

Figura 3: Interface com o usuário

O dispositivo Display de LCD é responsável por reproduzir as informações, como orientações e etapas, e fornecer a acessibilidade visual. Para conexão do display ao Raspberry Pi 2 foram utilizadas 6 portas GPIO. As conexões de saída do display referentes ao contraste e backlight são conectadas a dois potenciômetros de forma a controlar o brilho de background e intensidade da cor dos textos.
Para inicializar a programação é necessário pressionar o botão Menu. Nesta ação, o software acessa uma rotina que imprime no display LCD uma lista suspensa contendo todos os medicamentos cadastrados no banco de dados da Anvisa até a versão de software do Gerenciador de pílulas inteligente instalada. A lista de medicamentos pode ser examinada utilizado os botões de seta para cima e seta para baixo através de um sistema de rolagem.

Quando o medicamento listado é escolhido, deve ser pressionado o botão Menu (Sim). O software inicia uma rotina de busca pelo próximo compartimento desocupado no disco de compartimentos, rotacionando o disco até onde a seção disponível ficará acessível para inserção da pílula. Sucessivamente, o software pede para que seja confirmada a escolha.

$\mathrm{Na}$ etapa seguinte da rotina é programado o horário de ingestão da primeira dose através das setas para cima e para baixo, onde é possível navegar entre os valores de horas e minutos. O software irá solicitar o intervalo de ingestão das pílulas. Em seguida o protótipo irá procurar um novo compartimento disponível para que o usuário preencha com o medicamento, e assim sucessivamente, até o momento em que o usuário declare para o software que não deseja prosseguir com o abastecimento de pílulas para determinado tratamento.

Quando chega o horário em que deve ser ingerida alguma pílula é emitido um alarme visual e um alarme sonoro. O Alarme visual é composto de um diodo emissor de luz (LED), na cor azul. O alarme sonoro é composto por um dispositivo transdutor piezoelétrico de cerâmica, um buzzer. Esses alarmes continuam até que o usuário pressione a chave Menu (SIM) e interrompa a rotina, partindo para próxima etapa, que é a liberação do comprimido.

Para a visualização das informações inseridas pelo usuário no momento das parametrizações e dos dados de funcionamento do Gerenciador Inteligente de Pílulas, é gerado um arquivo de extensão $\mathrm{CSV}$, constantemente atualizado. Esse arquivo é enviado à web através de armazenamento em nuvem. O serviço escolhido para armazenamento foi o Google Drive, que exige login e senha, através de uma conta já cadastrada no Google.

\subsection{Case}

Para armazenar os componentes do dispositivo, foi desenvolvido um case com um design simples que favorece a utilização do mesmo. Possui largura de $17 \mathrm{~cm}$, comprimento de $18,5 \mathrm{~cm}$ e altura de $19,7 \mathrm{~cm}$. A Figura 4 apresenta o esboço do case.

\section{TESTES E RESULTADOS}

A Figura 5 apresenta o protótipo desenvolvido do Gerenciador de Pílulas Inteligente.

O módulo do motor de passo junto ao controle de posição apresentou respostas satisfatórias quando é inserido e gerenciado pílulas. Foi observado que, quando o horário de ingestão de pílulas coincide com o horário corrente, há uma latência mínima e esperada, devido ao tempo de execução de todas as rotinas, até o medicamento estar disponível para ingestão.

A precisão de posição do disco de compartimentos foi testada, avaliando erros de posição do disco de compartimentos, desde erros de milímetros, até erros que ocorressem a disponibilizar medicamentos errados, devido a falha do encoder. Foram coletadas 76 amostras 


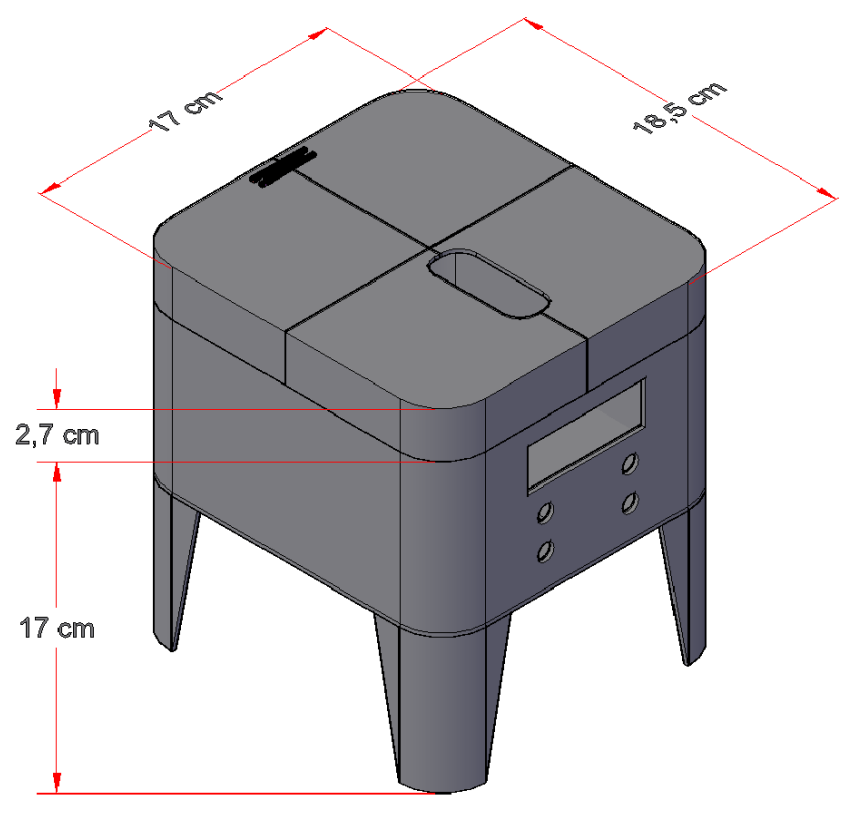

Figura 4: Esboço do case

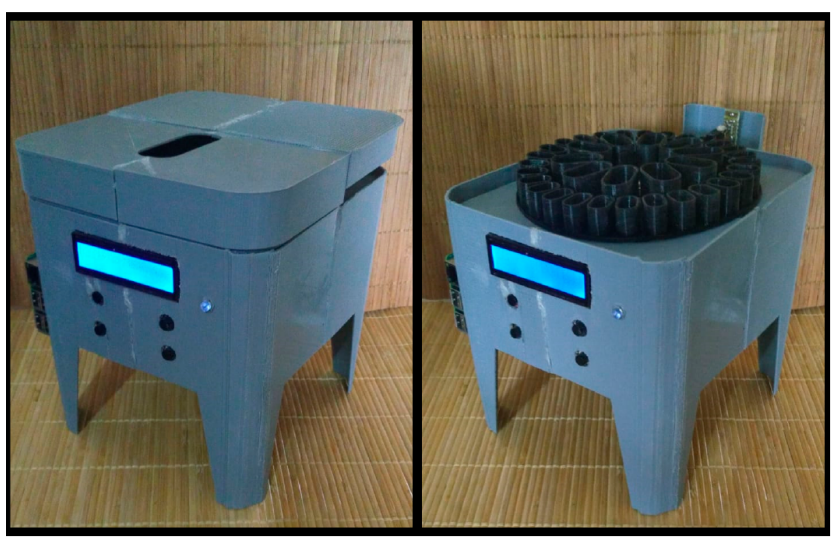

Figura 5: Protótipo do Gerenciador Inteligente de Pílulas

de posição considerando a programação do menu e a rotina de disponibilização de pílulas.

Foi possível verificar que existem pequenas variações na posição, o ponto de referência utilizado foi onde há os alçapões para disponibilizar as pílulas, gerando um desvio médio de $0,0263 \mathrm{~mm}$. Em virtude da margem de segurança de $1 \mathrm{~mm}$ das dimensões dos alçapões e as dimensões dos compartimentos, as amostragens de erros acima demonstradas se tornam irrelevantes para impedir que um medicamento seja dispensado. Valores superiores a $2 \mathrm{~mm}$ poderiam apresentar risco mínimo.

Os resultados do tempo de resposta de acesso ao menu e da programação, bem como da criação de relatórios acessados remotamente são considerados aceitáveis. Os botões da interface do usuário se mostraram práticos na funcionalidade do protótipo.

\section{CONCLUSÕES}

O Gerenciador de Pílulas Inteligente foi desenvolvido de forma a auxiliar e proporcionar qualidade de vida a idosos e cuidadores. Gerenciando, disponibilizando e emitindo relatórios, proporciona ao usuário uma gestão eficiente junto a automação e conforto. A partir da programação de horário dos medicamentos, o dispositivo realiza as atividades de gerenciar os horários e medicamentos sem necessidade de intervenção humana, e também alertar e disponibilizar ao usuário quando cada pílula deve ser ingerida.

O sistema de encoder, responsável por monitorar e controlar a posição do disco de compartimentos apresentou um Desvio médio de $0,0263 \mathrm{~mm}$, sendo os casos mais críticos com erro de $2 \mathrm{~mm}$, valores que tornam o sistema satisfatório para o tipo de solução proposta, e não acarretam erros mais graves, tal como sobreposição de pílulas. Foram utilizadas 78 amostras para chegar em dados estatísticos que comprovassem e averiguassem o desvio padrão de posição, quanto ao disco de posição, de acordo com os resultados, foi possível confirmar a eficiência do dispositivo. Quanto à disponibilização de pílulas nos horários programados, a taxa de erro foi nula.

\section{REFERÊNCIAS}

[1] Adriano dos Reis, Cristiano Pereira de Lima, Daniel Henrique Chessa de Lourena, and Luan Custório da Silva Araújo. 2012. Dispensador automático de medicamento. In Anais do IX Congresso Científico da UNIRP. São José do Rio Preto, 92-93.

[2] Lina Patrícia Navarro Carrasco. 2018. Dispensador automático de medicamentos sólidos - DIAMS. Thesis. Universidad Internacional de La Rioja (UNIR). https: //reunir.unir.net/handle/123456789/7209

[3] Rosana Polatto, Ana Cristina Maurício Ferreira, Wangner Barbosa da Costa, and Maria Aline Lemos Silva Thobias. 2017. TECNOLOGIA ASSISTIVA NO AUXÍlIO AO IDOSO: proposta de dispenser de comprimidos para uso doméstico. In Blucher Design Proceedings. Editora Blucher, São Paulo, 1806-1813. https://doi.org/10. 5151/16ergodesign- 0184

[4] Silvana Ramos. 2016. Dispensador eletrônico de medicamentos. https://www.behance.net/gallery/51732005/DIME-dispensador-eletr\{ô\}nico-demedicamentos-para-idoso

[5] André Simões, Leonardo Athias, and Luanda Botelho. 2018. Panorama nacional e internacional da produção de indicadores sociais : grupos populacionais específicos e uso do tempo (1(6) ed.). IBGE, Coordenação de População e Indicadores Sociais, Rio de Janeiro. 350 pages.

[6] Camila Figueiró Vasconcellos and Denise Milão. 2019. Hipodermóclise: alternativa para infusão de medicamentos em pacientes idosos e pacientes em cuidados paliativos. PAJAR - Pan American fournal of Aging Research 7, 1 (jun 2019), 32559. https://doi.org/10.15448/2357-9641.2019.1.32559 